\title{
Vulnerabilidad ante sismos y tsunami de las instituciones educativas ubicadas en la Provincia Constitucional del Callao
}

\author{
Vulnerability to earthquakes and tsunami of the educational institutions \\ located in the Constitutional Province of Callao
}

\author{
Maritza Guzmán Collazos ${ }^{1}$, Julio Jesús Quijano Vargas ${ }^{2}$
}

Recibido: 08/01/2020 - Aprobado: 05/12/2020 - Publicado: 18/06/2021

\begin{abstract}
RESUMEN
Conocida la condición de vulnerabilidad del Perú a enfrentar un evento sísmico de gran intensidad y en consecuencia un Tsunami, este estudio realiza un análisis del nivel de exposición de las Instituciones Educativas (IE) según su ubicación geográfica, para lo cual se utiliza la plataforma geoespacial Sistema de Información para la Gestión del Riesgo de Desastres (SIGRID), que alberga la información oficial, actualizada, técnico y científica producida en el Perú. La metodología implica dividir la provincia constitucional del Callao en 3 sectores delimitados a los que al hacer la superposición de capas de información para Microzonificación sísmica se obtuvo que de las 757 IE expuestas el $69 \%$ están ubicados en Zona II Depósitos de arena de compacidad media a densa y el $9 \%$ ubicados en zonas tipo IV y V Taludes inestables con fuerte pendiente y Depósitos de escombros y/o desechos respectivamente. Para el análisis frente a Tsunamis se obtuvo que de las $149 \mathrm{IE}$ ubicadas en zona de inundación el $31.5 \%$ serian inundados a consecuentes de un sismo de intensidad $8.5 \mathrm{Mv}$. y el $68.5 \%$ a consecuencia de un sismo de intensidad $9.0 \mathrm{Mv}$.
\end{abstract}

Palabras clave: Vulnerabilidad; Sismo; Tsunami; Instituciones Educativas; Callao.

\begin{abstract}
Known the vulnerability condition of Peru to face a seismic event of great intensity and consequently a Tsunami, this study makes an analysis of the level of exposure of the Educational Institutions (IE) according to their geographical location, for which geospatial platform is used SIGRID Information System for Disaster Risk Management, which houses the official and updated scientific and technical information produced in Peru. The methodology involves dividing La Provincia Constitutional del Callao into 3 delimited sectors to which when making the superposition of layers of information for seismic microzoning, it was obtained that of the 757 IE exposed, $69 \%$ are located in Zone II. dense and $9 \%$ located in zones type IV and V unstable slopes with steep slopes and deposits of debris and / or waste respectively. For the analysis against Tsunamis, it was obtained that of the $149 \mathrm{IE}$ located in the flood zone, $32 \%$ would be flooded due to an earthquake of $8.5 \mathrm{Mv}$ and $68 \%$ consequence to an earthquake of intensity $9.0 \mathrm{Mv}$.
\end{abstract}

Keywords: Vulnerability; Earthquake; Tsunami; Educational Institutions; Callao.

1 Ingeniera Ambiental CIP, Licenciada en Enfermería CEP, Bachiller en Ingeniería Ambiental, Bachiller en Enfermería, Maestrista en Sistemas integrados de Gestión en Seguridad, Salud en el trabajo y Medio Ambiente. Universidad Nacional Mayor de San Marcos, Lima, Perú.

E-mail: maritzagc654@gmail.com - ORCID: https://orcid.org/0000-0003-4657-1514

2 Ingeniero Mecánico de Fluidos CIP, Bachiller en Ingeniería Mecánica de Fluidos, Doctorando en Ciencias Ambientales Universidad Nacional Mayor de San Marcos. Lima, Perú. E-mail: jäv09@gmail.com - ORCID: https://orcid.org/0000-0001-6355-5052 


\section{INTRODUCCIÓN}

En el Perú se desarrolla continuamente un proceso de convergencia entre la placa de Nazca (Oceánica) que avanza por debajo de la Placa Sudamericana (Continental), lo cual genera la actual geodinámica y geomorfología del país. Esta dinámica genera sismos de diferentes magnitudes y focos, situados a diferentes profundidades, todos ellos a consecuencia de la fricción de las placas en mención, a la deformación cortical a niveles superficiales y a la deformación interna de la placa oceánica por debajo de la cordillera. Actualmente se dispone de catálogos sísmicos más completos, información geofísica de mayor confiabilidad, permitiendo conocer con mayor precisión la ubicación de las áreas que se originaría un nuevo terremoto en el futuro. Este es el caso de la región de la costa central del Perú, donde persiste la ausencia de un terremoto de gran magnitud desde el año 1746, y cuya acumulación de energía podría originar un evento sísmico de magnitud por encima a 8,5 Mw. (Instituto Geofisico del Peru, 2010).

Anualmente el Instituto Geofísico del Perú reporta entre 150 a 200 sismos percibidos los cuales presentaron intensidades mínimas de II-III Escala Mercali (MM) y magnitudes Escala Richter $(\mathrm{ML}) \geq 4$. A esta condición se suma el crecimiento desordenado de las ciudades construidas en zonas de alto peligro sísmico, ocupando áreas denominadas no urbanizables, exponiéndose al riesgo de sufrir pérdidas económicas, materiales, humanas en caso de presentarse un terremoto de gran intensidad (Instituto Geofisico del Peru, 2010).

Con respecto a los TSUNAMIS, el Dr. Tavera director del IGP sostiene: "El tsunami de mayor tamaño ocurrió después del sismo del 28 de octubre de 1746, de magnitud $\sim 8,6 \mathrm{Mw}$ y epicentro frente al departamento de Lima. Media hora después de ocurrido el evento sísmico, se generó un gran tsunami que golpeó la costa de la región central del Perú causando la destrucción absoluta del puerto. El Callao fue impactado por olas de 24 metros que inundaron distancias de hasta $5 \mathrm{~km}$. De los 23 barcos anclados en el puerto, 19 fueron hundidos y cuatro trasladados tierra dentro. En total, las víctimas del terremoto y tsunami alcanzaron el número de 4000 personas. En el puerto solo quedaron cascajos y arenas que se depositaron sobre los escombros dejados por el sismo y tsunami” (Tavera Hernando, 2014).

Conocida la potencial ocurrencia de sismos y tsunamis a fin de advertir las consecuencias asociadas, los escenarios de riesgo son construidos desde la identificación $\mathrm{y}$ análisis de los dos factores que conforman el riesgo: los peligros (componente extrínseco) y las vulnerabilidades (componente intrínseco) y a su vez la interacción de estos en el lugar de estudio (PLANAGERD 2021, 2014).

En nuestro país, la vulnerabilidad física a nivel de infraestructura y equipamiento de las instituciones educativas es fundamentalmente alta, principalmente en las zonas rurales y con menor disponibilidad de recurso económicos. Un número considerable de las instituciones educativas no cuentan con condiciones necesarias para afrontar las consecuencias de las emergencias y desastres que podrían ocurrir, ni para reducir los impactos negativos que podrían afectar al alumnado (Ministerio de Educación, 2015).
Las instituciones educativas albergan durante al menos 05 horas diarias menores de edad, quienes son entregados por sus padres a los maestros y directivos suponiendo que durante ese tiempo sus hijos estarían a salvo, sin embrago para el caso de algunas IE ubicadas en la provincia constitucional del Callao por sus condiciones geográficas particulares conviven con riesgos internos debido la falta de mantenimiento de las edificaciones, construcción ajenas a las Normas Sismo resistentes por su antigüedad, disminuida cultura de prevención a ello presentan en adición el riesgo externo de enfrentar sismos de gran intensidad y consecuente Tsunamis.

Considerando el riesgo de sismos y tsunamis a los que están expuestas las I.E. ubicadas en la mencionada provincia costera, este estudio propone una metodología de fácil aplicación que permite visualizar escenarios de riesgo para sismos y tsunamis considerando variables fundamentales como los relacionados a los parámetros de calidad de suelo, ubicación de las instituciones educativas, el nivel de sismo las potenciales zonas de inundación a consecuencia de tsunamis posterior a un evento sísmico que podrían ocurrir en la provincia constitucional de Callao; a fin de brindar información específica que servirá como herramienta de decisión a las autoridades competentes.

\section{MÉTODOS}

\subsection{Material}

- Bibliográfico: Técnico científico generado por instituciones públicas y privadas, principalmente investigaciones en relación con sismología y presencia de Tsunamis.

- Programa Excel ${ }^{\circledR}$. Para análisis, procesamiento e interpretación de datos

- Plataforma geoespacial en la web SIGRID CENEPRED

SIGRID es un sistema de información geográfico en línea, diseñado para el uso masivo de la población, que brinda información geoespacial técnico científico y registros documentarios en relación al riesgo de desastres, a través de herramientas y funcionalidades que permitan consultar, acceder, monitorear, analizar, procesar modelos, cargar y descargar información específica que contribuya con el planeamiento y formulación de proyectos de inversión pública asociados a la estimación, prevención, reducción del riesgo de desastres y la reconstrucción (CENEPRED, 2020).

\subsection{Métodos}

Por fines didácticos diferenciamos la Provincia constitucional del Callao en 03 sectores.

\footnotetext{
- Sector Norte: Latitud S $11^{\circ} 48^{\prime} 00^{\prime \prime}-11^{\circ} 56^{\prime} 00^{\prime \prime}$

- Sector Centro: Latitud S $11^{\circ} 56^{\prime} 00^{\prime \prime}-12^{\circ} 01^{\prime} 00^{\prime \prime}$

- Sector Sur: Latitud S $12^{\circ} 01^{\prime} 00^{\prime \prime}-12^{\circ} 05^{\prime} 00^{\prime \prime}$
} 
a. Para laidentificación deIE según su microzonificación sísmica se realiza la siguiente superposición de capas de información:

- Elementos expuestos / Instituciones educativas / públicos privadas

- Cartografía de peligros / geodinámica interna / Zonificación sísmica / Microzonificación sísmica Cismid.

- $\quad$ En cada sector se contabiliza las IE públicas y privadas ubicadas en cada microzonificación sísmica.

- Construimos tabla de datos y analizamos los resultados

- Para identificar la microzonificación utilizaremos la Tabla 1 Microzonificación sísmica.

b. Para la identificación de IE ubicadas en zona de inundación según el nivel de sismos al que sería expuesto se realiza la superposición de capas de información

- Elementos expuestos / Instituciones educativas / públicos privadas

- Cartografía de peligros / geodinámica interna / Tsunamis

- En cada sector se contabiliza las IE públicas y privadas ubicadas en zona de inundación a consecuencia del nivel de sismo correspondiente.
- Construimos tabla de datos y analizamos los resultados

- Para identificar la zona de inundación por tsunami utilizaremos la Tabla 2, Zona de inundación por tsunami.

\section{RESULTADOS Y DISCUSIÓN}

Análisis de los resultados, ubicación de las IE públicas y privadas según la microzonificación sísmica y zona de inundación por tsunami de cada sector:

\section{A. Sector Norte}

En la Figura 1 y Tabla 3 se observa que de las 239 IE ubicadas en el sector norte el $65.3 \%$ se encuentra en la Zona II - Correspondiente a depósitos de arena de compacidad media a densa, mientras que el $0.4 \%$ y $2.9 \%$ se encuentran en las zonas IV y V correspondiente a taludes inestables con fuerte pendiente y depósitos de escombros $\mathrm{y} / \mathrm{o}$ desechos respectivamente.

En la Figura 2 y Tabla 4 se observa que de las 17 IE expuestas a inundación ante potencial Tsunami el 11.8\% serian inundados posteriormente a la presencia de un sismo de intensidad $8.5 \mathrm{Mv}$ y el $88.2 \%$ en consecuencia de un sismo de intensidad 9.0 Mv.

\section{B. Sector Centro:}

En la Figura 3 y Tabla 5 se observa que de las 107 IE ubicadas en el sector centro el $72 \%$ se encuentra en la Zona II - Correspondiente a depósitos de arena de compacidad

Tabla 1. Microzonificación sísmica

\begin{tabular}{cl}
\hline Zonas & \multicolumn{1}{c}{ Microzonificación Sísmica } \\
\hline I & $\begin{array}{l}\text { Zonas de afloramiento de roca con diversos grados de fracturación, depósitos de grava y arena de compacidad densa a muy densa, } \\
\text { depósitos de limos y arcillas de consistencia rígida a muy rígida. Períodos de vibración ambiental menores a 0.30 s } \\
\text { Depósitos de arena de compacidad media a densa o arcillas y limos de consistencia media. Períodos de vibración ambiental menores } \\
\text { a } 0.40 \mathrm{~s}\end{array}$ \\
II & $\begin{array}{l}\text { Depósitos de arenas de compacidad suelta a media, depósitos de limos y arcillas de consistencia blanda a media. Períodos de vibración } \\
\text { ambiental mayores a } 0.40 \mathrm{~s} \text {. } \\
\text { III }\end{array}$ \\
Taludes inestables con fuerte pendiente, canteras informales, depósitos de suelos pantanosos, depósitos de arenas eólicas de \\
compacidad suelta potencialmente licuables. Zonas con alta amplificación sísmica. \\
Depósitos de escombros y/o desechos, rellenos antrópicos en el interior de antiguas excavaciones mineras.
\end{tabular}

Elaboración propia. Fuente (CENEPRED, 2020)

Tabla 2. Zona de inundación por tsunami

\begin{tabular}{cc}
\hline Zona de inundación por Tsunami & Descripción \\
\hline Intensidad de Sismo & Zona de inundación en consecuencia de un sismos de $8.5 \mathrm{Mv}$ \\
\hline $8.5 \mathrm{Mv}$ & Zona de inundación en consecuencia de un sismos de $8.5 \mathrm{Mv}$ \\
\hline Elaboración propia. Fuente (CENEPRED, 2020)
\end{tabular}




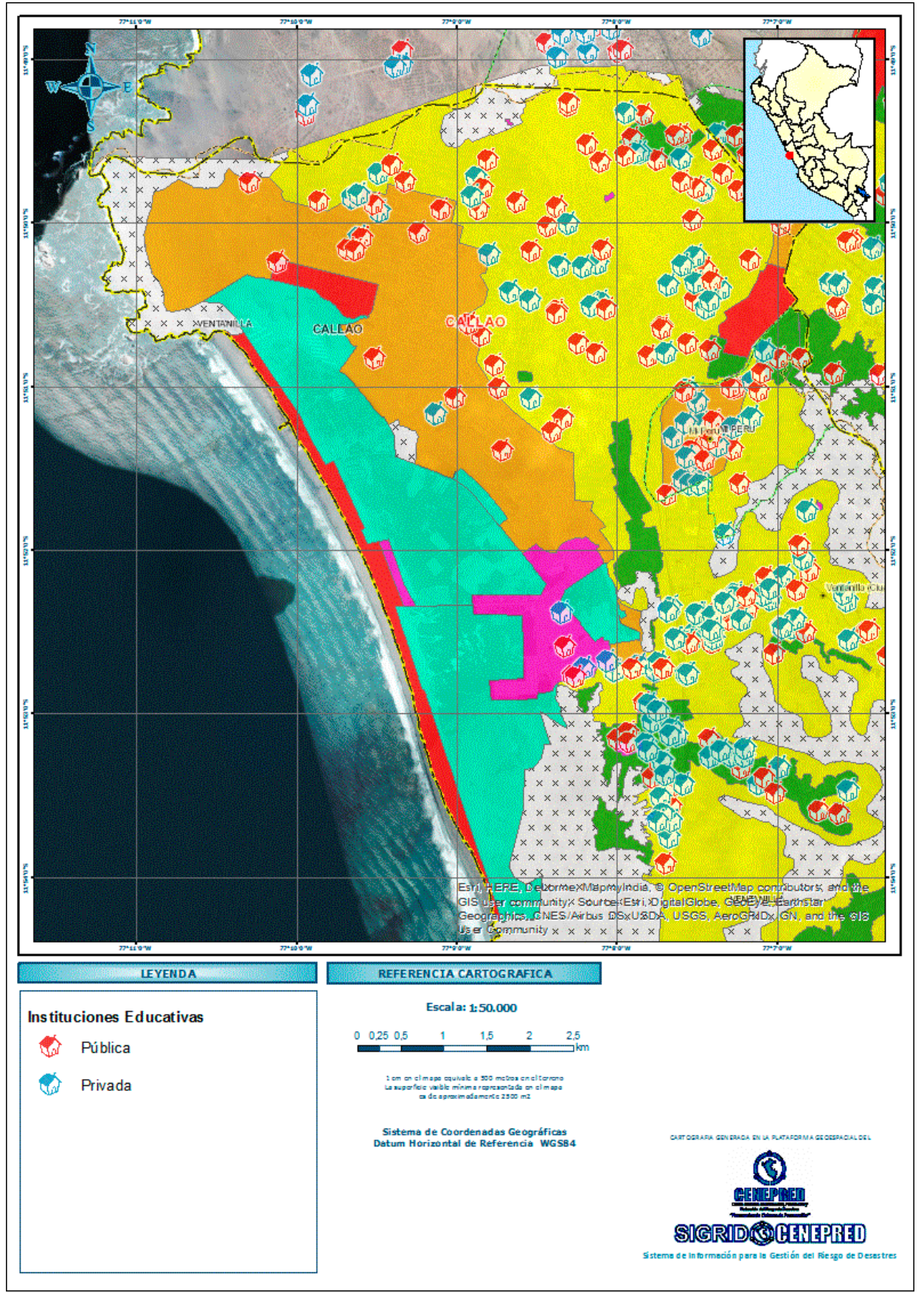

Figura 1. Ubicación de IE según Microzonificación sísmica - Sector Norte Elaboración propia -Fuente: SIGRID

Tabla 3. Ubicación detallada de IE según Microzonificación sísmica - Sector Norte

\begin{tabular}{|c|c|c|c|c|c|}
\hline \multicolumn{6}{|c|}{ Instituciones Educativas } \\
\hline Sector & Zona Sísmica & Públicas & Privadas & Total & Porcentaje (\%) \\
\hline \multirow{6}{*}{ Norte } & 1 & 20 & 22 & 42 & 17.6 \\
\hline & $\|$ & 77 & 79 & 156 & 65.3 \\
\hline & III & 11 & 22 & 33 & 13.8 \\
\hline & IV & 1 & 0 & 1 & 0.4 \\
\hline & V & 4 & 3 & 7 & 2.9 \\
\hline & Total & 113 & 126 & 239 & 100 \\
\hline
\end{tabular}

Elaboración Propia 


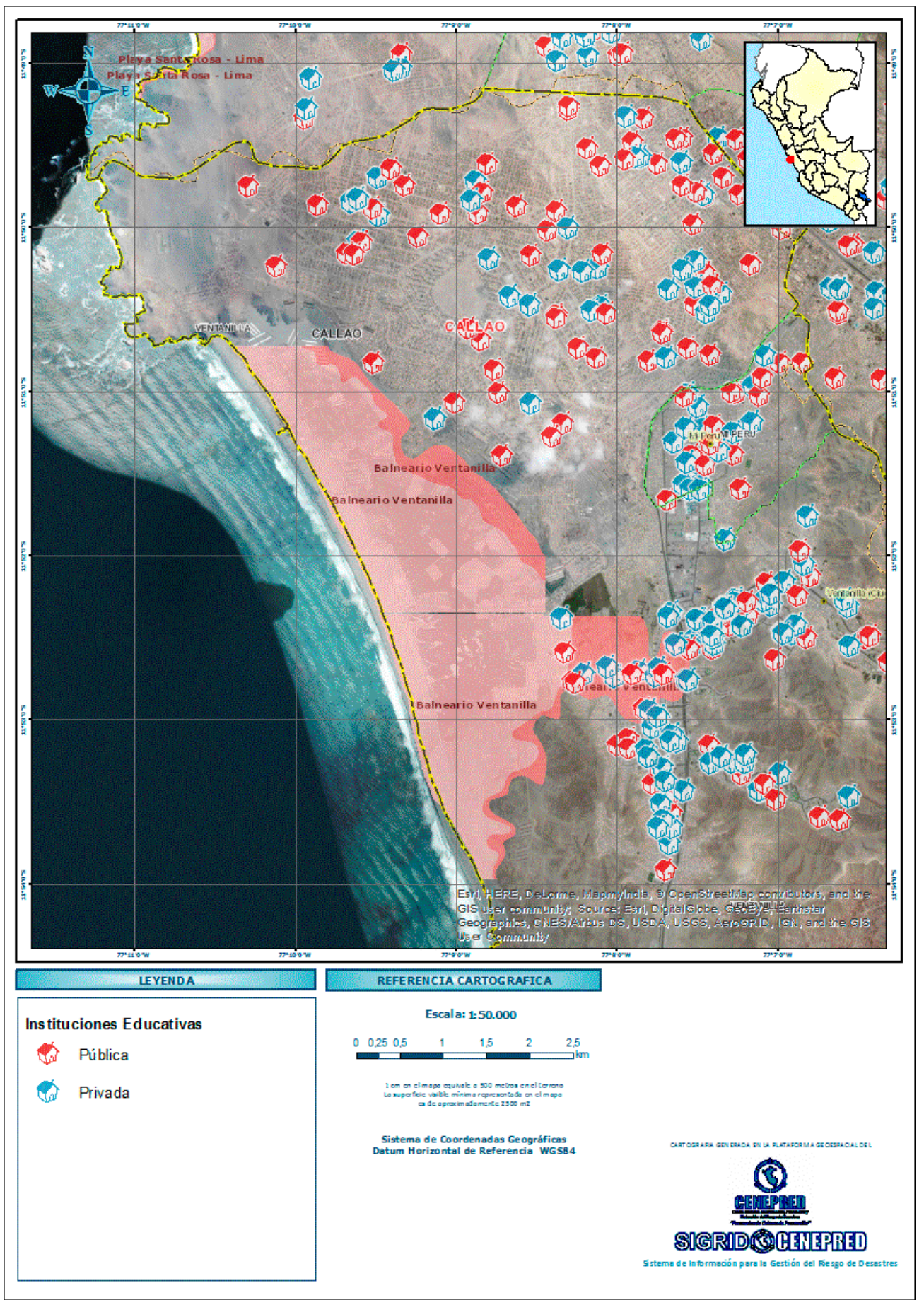

Figura 2. Ubicación de IE ante Potencial Tsunami - Sector Norte

Elaboración propia -Fuente SIGRID

Tabla 4. Cantidad IE expuestas a inundación ante Potencial Tsunami - Sector Norte

\begin{tabular}{cccccc}
\hline \multicolumn{5}{c}{ Instituciones Educativas } \\
\hline Sector & Intensidad Sismo & Públicas & Privadas & Total & Porcentaje (\%) \\
\hline \multirow{3}{*}{ Norte } & $8.5 \mathrm{Mv}$ & 1 & 1 & 2 & 11.8 \\
& $9.0 \mathrm{Mv}$ & 5 & 10 & 15 & 88.2 \\
& Total & 6 & 11 & 17 & 100.0 \\
\hline
\end{tabular}

Elaboración Propia 


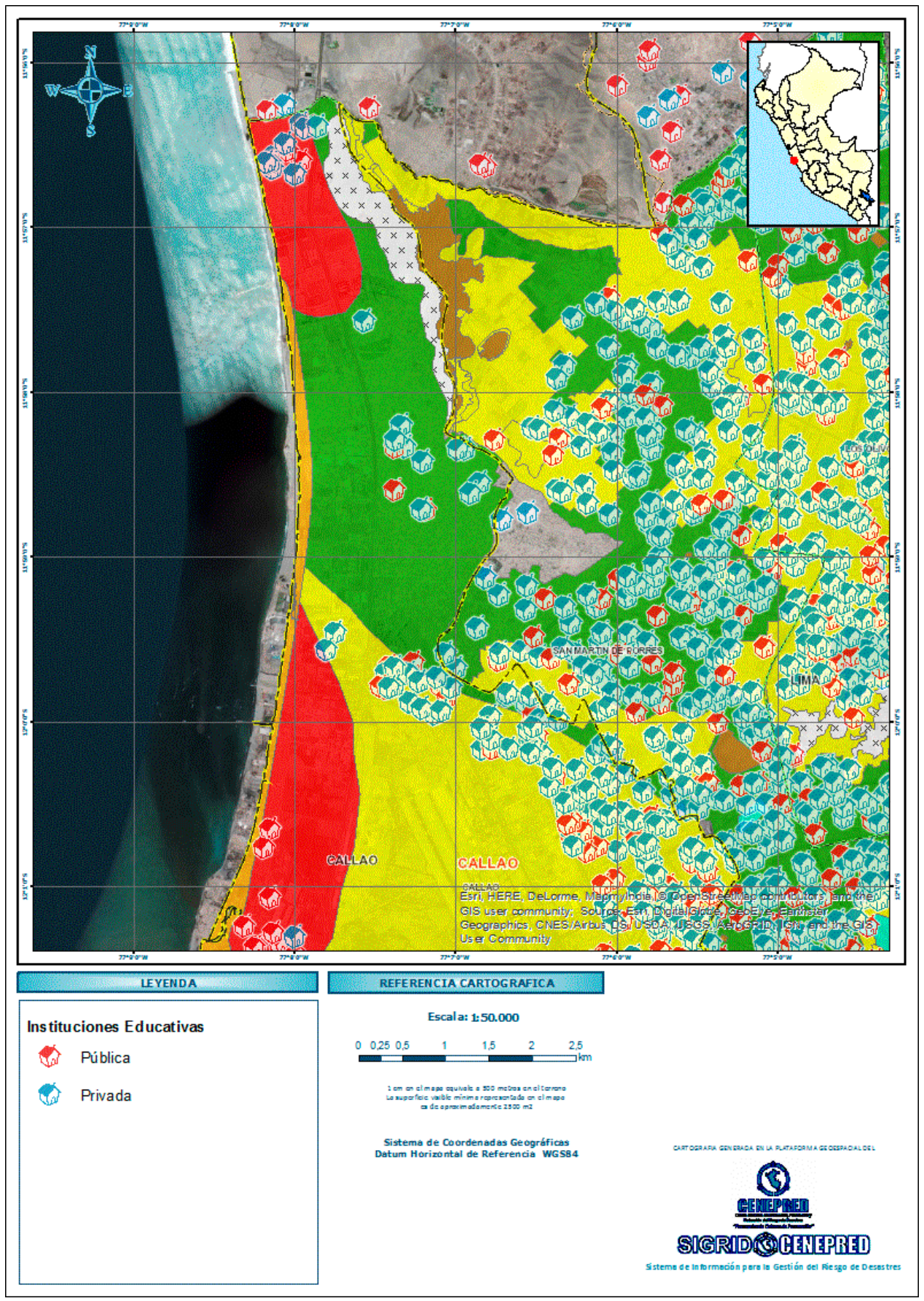

Figura 3. Ubicación de IE según Microzonificación sísmica - Sector Centro Elaboración propia -Fuente SIGRID

Tabla 5. Ubicación detallada de IE según Microzonificación sísmica - Sector Centro

\begin{tabular}{cccccc}
\hline & \multicolumn{2}{c}{ Instituciones Educativas } \\
\hline Sector & Zona Sísmica & Públicas & Privadas & Total & Porcentaje (\%) \\
& I & 4 & 12 & 16 & 15.0 \\
& II & 21 & 56 & 77 & 72.0 \\
\multirow{3}{*}{ Centro } & III & 0 & 0 & 0 & 0.0 \\
& IV & 5 & 7 & 12 & 11.2 \\
& V V & 2 & 0 & 2 & 1.9 \\
& Total & 32 & 75 & 107 & 100.0 \\
\hline
\end{tabular}


media a densa, mientras que el $11.2 \%$ y $1.9 \%$ se encuentran en las zonas IV y V correspondiente a taludes inestables con fuerte pendiente y depósitos de escombros y/o desechos respectivamente.

En la Figura 4 y Tabla 6 se observa que de las $20 \mathrm{IE}$ expuestas a inundación ante potencial Tsunami el 35\% serian inundados posteriormente a la presencia de un sismo de intensidad $8.5 \mathrm{Mv}$ y el $65 \%$ en consecuencia de un sismo de intensidad 9.0 Mv.

\section{Sector Sur}

En la Figura 5 y Tabla 7 se observa que de las 411 IE ubicadas en el sector sur el $69.3 \%$ se encuentra en la Zona II - Correspondiente a depósitos de arena de compacidad

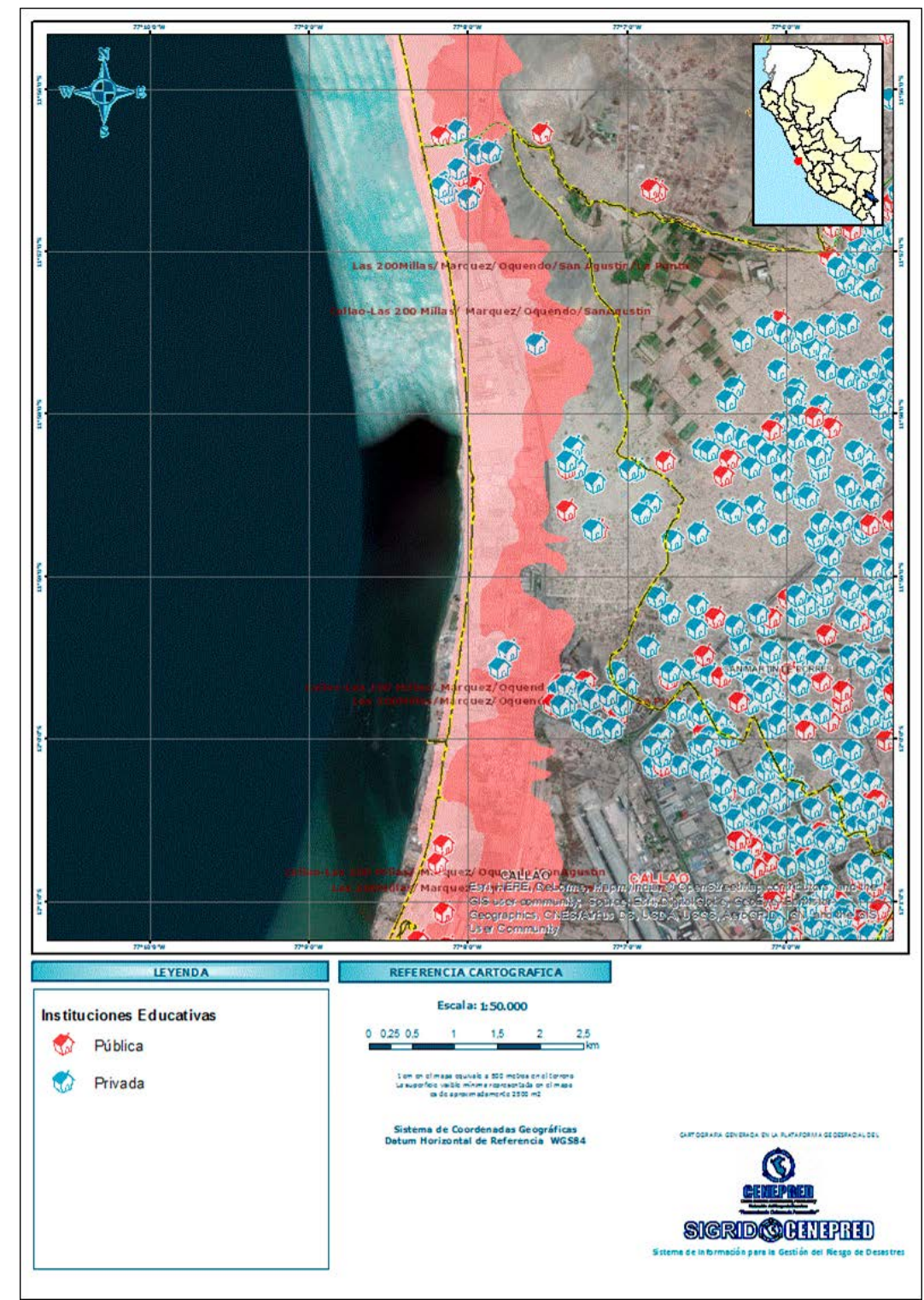

Figura 4. Ubicación de IE ante Potencial Tsunami - Sector Norte

Elaboración propia -Fuente SIGRID

Tabla 6. Cantidad IE expuestas a inundación ante Potencial Tsunami - Sector Centro

\begin{tabular}{cccccc}
\hline \multicolumn{5}{c}{ Instituciones Educativas } \\
\hline Sector & Intensidad Sismo & Públicas & Privadas & Total & Porcentaje (\%) \\
& $8.5 \mathrm{Mv}$ & 3 & 4 & 7 & 35 \\
Centro & $9.0 \mathrm{Mv}$ & 3 & 10 & 13 & 65 \\
& Total & 6 & 14 & 20 & 100 \\
\hline
\end{tabular}

Elaboración Propia 


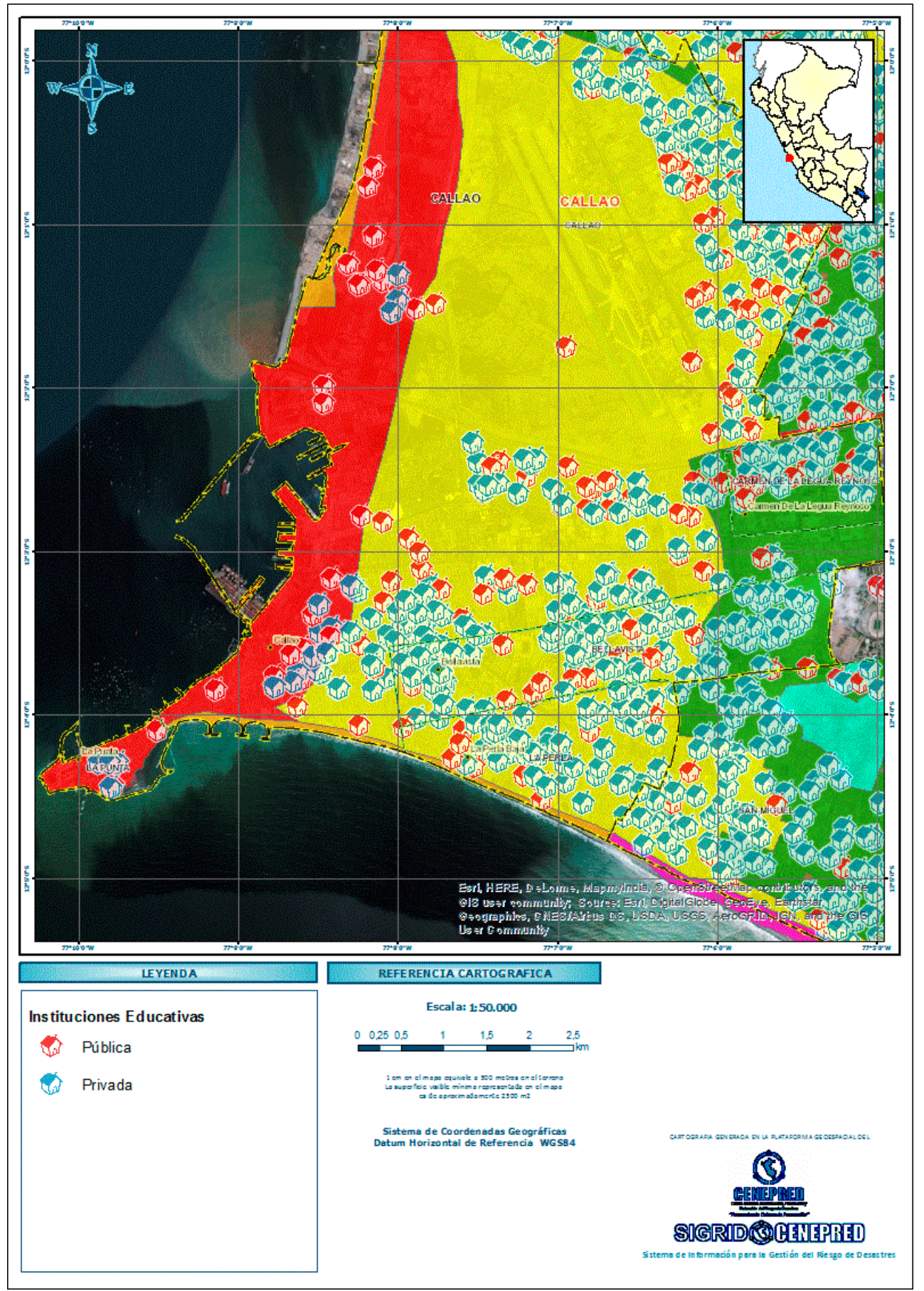

Figura 5. Ubicación de IE según Microzonificación sísmica - Sector Sur

Elaboración propia -Fuente SIGRID

Tabla 7. Ubicación detallada de IE según Microzonificación sísmica - Sector Sur

\begin{tabular}{|c|c|c|c|c|c|}
\hline \multicolumn{6}{|c|}{ Instituciones Educativas } \\
\hline Sector & Zona Sísmica & Públicas & Privadas & Total & Porcentaje (\%) \\
\hline \multirow{6}{*}{ Sur } & 1 & 15 & 65 & 80 & 19.5 \\
\hline & $\|$ & 85 & 200 & 285 & 69.3 \\
\hline & III & 0 & 0 & 0 & 0.0 \\
\hline & IV & 21 & 25 & 46 & 11.2 \\
\hline & V & 0 & 0 & 0 & 0.0 \\
\hline & Total & 121 & 290 & 411 & 100.0 \\
\hline
\end{tabular}

Elaboración Propia 
media a densa, mientras que el $11.2 \%$ se encuentran en la zona IV correspondiente a taludes inestables con fuerte pendiente.

En la Figura 6 y Tabla 8 se observa que de las 212 IE expuestas a inundación ante potencial Tsunami el 33.9\% serian inundados posteriormente a la presencia de un sismo de intensidad $8.5 \mathrm{Mv}$ y el $66.1 \%$ en consecuencia de un sismo de intensidad 9.0 Mv.

\section{Los 03 sectores}

Para el escenario de sismos

En la Figura 9 se observa que de las 757 IE ubicadas en los 03 sectores de la provincia constitucional del Callao, el $68.4 \%$ se encuentra en la Zona II - Correspondiente a depósitos de arena de compacidad media a densa, mientras que el $1.2 \%$ y $7.8 \%$ se encuentran en las zonas IV y V

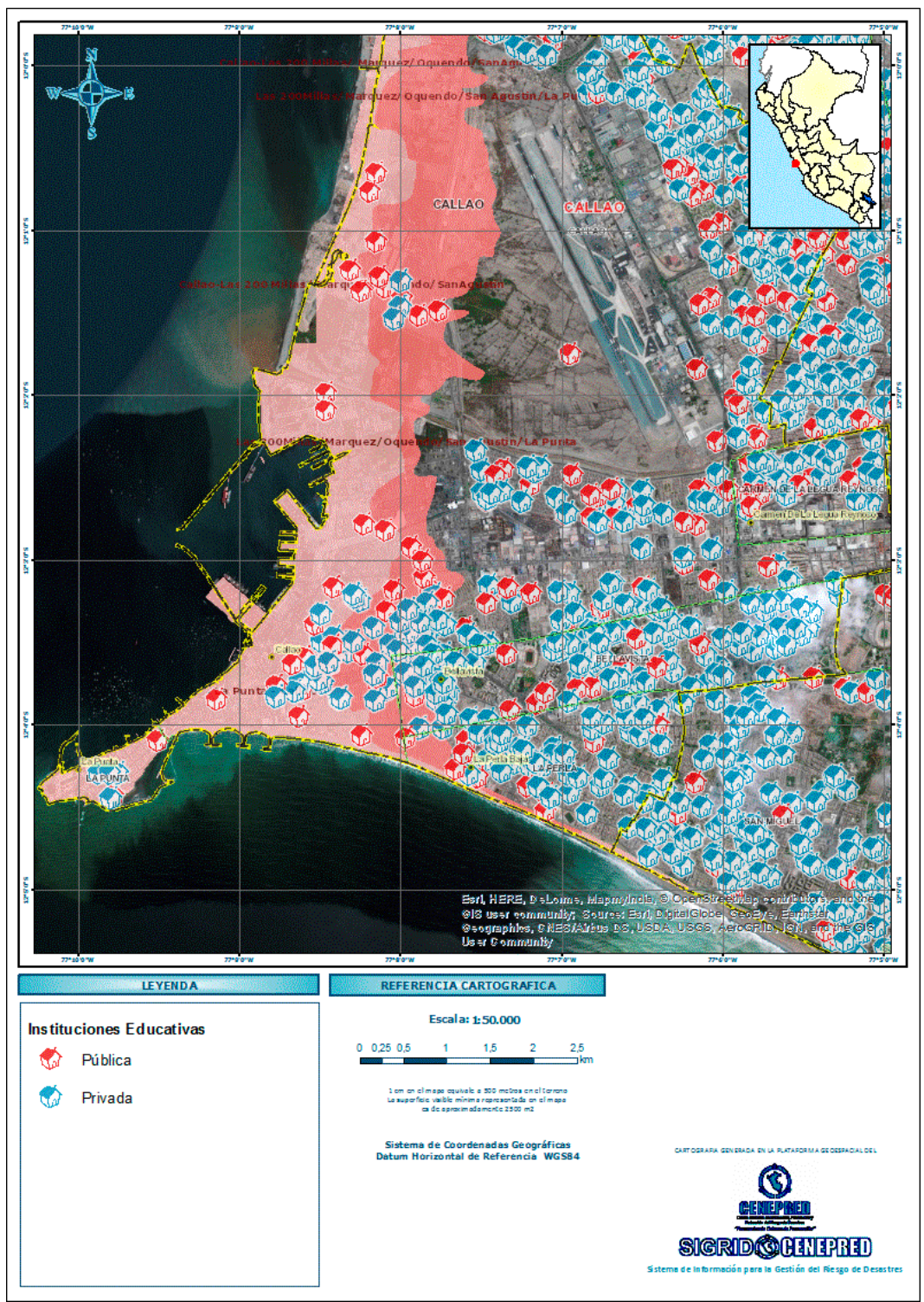

Figura 6. Ubicación de IE ante Potencial Tsunami - Sector Norte Elaboración propia -Fuente SIGRID

Tabla 8. Cantidad IE expuestas a inundación ante Potencial Tsunami - Sector Sur

\begin{tabular}{cccccc}
\hline \multicolumn{5}{c}{ Instituciones Educativas } \\
\hline \multirow{3}{*}{ Sector } & Intensidad Sismo & Públicas & Privadas & Total & Porcentaje (\%) \\
& $8.5 \mathrm{Mv}$ & 18 & 20 & 38 & 33.9 \\
Sur & $9.0 \mathrm{Mv}$ & 22 & 52 & 74 & 66.1 \\
& Total & 40 & 72 & 112 & 100.0 \\
\hline
\end{tabular}

Elaboración propia 
correspondiente a taludes inestables con fuerte pendiente y depósitos de escombros y/o desechos respectivamente.

Tabla 9. Ubicación detallada de IE según Microzonificación sísmica Ubicación de IE de la provincia constitucional del Callao según tipos de suelo

\begin{tabular}{ccc} 
Microzonificación & Numero de IE & Porcentaje (\%) \\
\hline I & 138 & 18.2 \\
\hline II & 518 & 68.4 \\
III & 33 & 4.4 \\
IV & 59 & 7.8 \\
V & 9 & 1.2 \\
\hline Total & 757 & 100.0 \\
\hline
\end{tabular}

Elaboración Propia

De los 757 IE ubicadas en la provincia constitucional del Callao $149(20 \%)$ se encuentran ubicadas en área de inundación por Tsunami a consecuencia de un potencial evento sísmico de $8.5 \mathrm{Mv}$ y $9.0 \mathrm{Mv}$, sobre estos casos los resultados de la superposición de capas de información permiten determinar lo siguiente:

Para el escenario de tsunamis

Tabla 10. Cantidad IE expuestas a inundación ante Potencial Tsunami

\begin{tabular}{ccc}
\hline \multicolumn{3}{c}{ Ubicación de IE de la provincia constitucional del Callao } \\
ante Tsunami \\
\hline Intensidad de Sismo & Numero de IE & Porcentaje (\%) \\
\hline $8.5 \mathrm{Mv}$ & 47 & 31.5 \\
$9.0 \mathrm{Mv}$ & 102 & 68.5 \\
Total & 149 & 100.0 \\
\hline
\end{tabular}

Elaboración Propia

En la Figura 9 se observa que de las 149 IE expuestas a inundación ante potencial Tsunami el $31.5 \%$ serian inundados posteriormente a la presencia de un sismo de intensidad $8.5 \mathrm{Mv}$ y el $68.5 \%$ en consecuencia de un sismo de intensidad 9.0 Mv.

\section{CONCLUSIONES}

- Las provincia constitucional del Callao presenta diversos elementos en la composición de sus suelos esta variedad da origen a la existencia de las cinco zonificaciones sísmicas en esta área geográfica (Tavera \& Burforn, 1998).

- En la Microzonificación sísmica de la Provincia constitucional del Callao delimitada en tres sectores (Norte, Centro y Sur) se obtuvo que de las 757 IE expuestas el 69\% están ubicados en la Microzonificación II correspondientes a depósitos de arena de compacidad media a densa y el $9 \%$ de las IE ubicados en Microzonificación tipo IV y $\mathrm{V}$ corresponden a taludes inestables con fuerte pendiente y depósitos de escombros y/o desechos respectivamente.

- Para el análisis frente a Tsunamis se obtuvo que de las 149 IE ubicadas en zonas expuestas a inundación ante potencial Tsunami en la provincia constitucional del Callao el $31.5 \%$ serian inundados posteriormente a la presencia de un sismo de intensidad $8.5 \mathrm{Mv}$ y el $68.5 \%$ en consecuencia de un sismo de intensidad 9.0 Mv.

- De los 757 IE ubicadas en la provincia constitucional del Callao 149 (20\%) se encuentran ubicadas en área de inundación por Tsunami a consecuencia de un potencial evento sísmico de 8.5 Mv y $9.0 \mathrm{Mv}$.

- Más del 50\% de las I.E entre públicas y privadas de la provincia constitucional del Callao se encuentran ubicadas en áreas que ponen en peligro a sus usuarios, si a ello sumamos la falta de mantenimiento de las edificaciones, la construcción de IE ajenas a las Normas Sismorresistentes, y la disminuida cultura de prevención de la población en general, solo estamos acumulando factores de riesgo que a corto o largo plazo terminarán costando mucho más que lo que se hubiese invertido en prevención de las pérdidas en todos los niveles.

- El área de Gestión de riesgo de desastres de la provincia constitucional del Callao deberá realizar la Evaluación de Riesgos (EVAR) a cada institución educativa analizada, considerando los resultados de este estudio.

\section{AGRADECIMIENTOS}

Al Centro Nacional de Estimación, Prevención y Reducción del Riesgo de Desastres por su esfuerzo en sistematizar la producción en investigación técnicocientífica del Perú y acercarlo de manera didáctica a la población en general.

\section{REFERENCIAS}

CENEPRED. (2020). Sistema de Información para la Gestión del Riesgo de Desastres - SIGRID. SIGRID. http://cenepred. gob.pe/web/

Instituto Geofisico del Peru. (2010). Atlas de peligros del Perú. SINPAD. http://sinpad.indeci.gob.pe/PortalSINPAD/ Default.aspx?ItemId=272

Ministerio de Educación. (2015). Plan de Gestión del Riesgo de Desastres en instituciones educativas. Ministerio de Educacion, 56. http://www.minedu.gob.pe/fenomeno-elnino/pdf/guia-plan-de-gestion-de-riesgo-2015.pdf

PLANAGERD 2021. (2014). Plan Nacional de Gestión del Riesgo de Desastres. Presidencia Del Consejo de Ministros. http://www.pcm.gob.pe/wp-content/uploads/2018/01/ PLANAGERD.pdf 
Tavera, H., \& Burforn, E. (1998). Sismicidad y sismotectónica de Perú. Física de La Tierra, 10, 187-220. https://doi. org/10.5209/rev_FITE.1998.n10.12999

Tavera Hernando. (2014). EVALUACION DEL PELIGRO ASOCIADOALOS SISMOS YEFECTOS SECUNDARIOS EN EL PERU 2014 - IGP. In Instituto Geofisico del Perú (Vols. 2014-11704, Issues 978-612-45795-9-2). https:// repositorio.igp.gob.pe/handle/IGP/777 
